\title{
Gastrointestinal symptoms in invasive pneumococcal disease: a cohort study
}

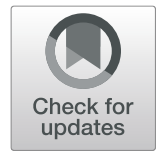

Hans Kristian Floeystad 1*, Jacob Dag Berild², Bjoern Jardar Brandsaeter ${ }^{3}$, Didrik Frimann Vestrheim², Dag Berild ${ }^{4,5}$ and Are Martin Holm ${ }^{5,6}$

\begin{abstract}
Background: The study aimed to assess whether gastrointestinal (Gl) symptoms at admission are associated with increased short-term mortality in patients with invasive pneumococcal disease (IPD).

Methods: We included all patients with IPD at Aker University Hospital in Oslo, Norway, from 1993 to 2008. Clinical data were registered. Survival data were retrieved from official registries. We used Cox regression and Kaplan-Meier curve to compare mortality within 28 days of admission in patients with and without Gl symptoms.

Results: Four hundred sixteen patients were included. Of these, 108 patients (26\%) presented with Gl symptoms, and 47 patients $(11 \%)$ with Gl symptoms only. Patients with $\mathrm{Gl}$ symptoms were younger $(p<0.001)$ and had less cardiovascular disease $(p<0.001)$, pulmonary disease $(p=0.048)$, and cancer $(p=0.035)$ and received appropriate antibiotic treatment later. After adjusting for risk factors, we found an increased hazard ratio of $2.28(95 \% \mathrm{Cl} 1.31-$ 3.97) in patients presenting with Gl symptoms. In patients with Gl symptoms only there was an increased hazard ratio of 2.24 (95\% Cl 1.20-4.19) in univariate analysis, which increased to 4.20 (95\% Cl $2.11-8.39)$ after multivariate adjustment. Fewer patients with Gl symptoms only received antibiotics upon admission.
\end{abstract}

Conclusions: A large proportion of IPD patients present with Gl symptoms only or in combination with other symptoms. Gl symptoms in IPD are associated with increased short-term mortality.

Keywords: Invasive pneumococcal disease, Sepsis, Pneumococcal bacteremia, Gastrointestinal symptoms, Mortality

\section{Background}

Streptococcus pneumoniae is a major cause of mortality and morbidity in adults [1-3]. Invasive pneumococcal disease (IPD), where S. pneumoniae is found in sterile sites like blood or spinal fluid, is the most severe form of pneumococcal disease with mortality rates of up to $20 \%$ $[4,5]$. These patients most often present with symptoms from the pulmonary system, like cough, dyspnea and chest pain combined with fever and chills, but some patients present with gastrointestinal (GI) symptoms such as acute abdominal pain [6], nausea and vomiting [7].
There are also case reports of IPD presenting to hospital with GI symptoms where many have been diagnosed as abdominal or pelvic infections [8-11].

Early detection of IPD is essential because of the high mortality during the first $48 \mathrm{~h}$, and some predictors of a fatal outcome have been identified [12, 13]. To our knowledge, there are no large studies comparing mortality in IPD patients presenting with GI symptoms to patients presenting without GI symptoms. We hypothesize that patients presenting with GI symptoms may have a higher mortality, possibly due to a diagnostic delay as a result of atypical presentation.

\footnotetext{
* Correspondence: hafloe@sshf.no

${ }^{1}$ Department of Internal Medicine, Sorlandet Hospital, Kristiansand, Norway

Full list of author information is available at the end of the article
}

C C The Author(s). 2020 Open Access This article is licensed under a Creative Commons Attribution 4.0 International License, which permits use, sharing, adaptation, distribution and reproduction in any medium or format, as long as you give appropriate credit to the original author(s) and the source, provide a link to the Creative Commons licence, and indicate if changes were made. The images or other third party material in this article are included in the article's Creative Commons licence, unless indicated otherwise in a credit line to the material. If material is not included in the article's Creative Commons licence and your intended use is not permitted by statutory regulation or exceeds the permitted use, you will need to obtain permission directly from the copyright holder. To view a copy of this licence, visit http://creativecommons.org/licenses/by/4.0/ The Creative Commons Public Domain Dedication waiver (http://creativecommons.org/publicdomain/zero/1.0/) applies to the data made available in this article, unless otherwise stated in a credit line to the data. 


\section{Methods}

\section{Study design}

Using a paper-based standardized data collection form we retrieved demographic, clinical, laboratory and microbiological data from hospital charts of all adult patients admitted to Aker University Hospital (AUH) between 1 January 1993 and 31 December 2008 with growth of S. pneumonia in blood or spinal fluid, as previously described [4]. During the inclusion period, AUH served an unselected catchment population of about 150,000 adults. The first 3 years, the data were collected retrospectively, and the rest were collected prospectively. If a patient had multiple hospitalizations for IPD, we included only the first hospitalization in this study.

\section{Antibiotic guidelines}

The national antibiotic guidelines for communityacquired pneumonia recommended benzylpenicillin 1,2 $\mathrm{g} \times 4$, and for septic patients benzylpenicillin $3 \mathrm{~g} \times 4$ plus gentamicin $5-7 \mathrm{mg} / \mathrm{kg}$.

\section{Data collection}

Blood cultures were routinely drawn in patients with a suspected infection and a body temperature higher than $38.5^{\circ} \mathrm{C}$, and in patients with a lower body temperature when a severe infection was suspected. A spinal tap was performed if a central nervous infection was suspected. The included patients had IPD, defined as growth of $S$. pneumonia in either blood culture or spinal fluid. Positive cultures were serotyped at the Norwegian Institute of Public Health (NIPH) by the Quellung reaction using serotype-specific antisera (Statens Seruminstitut, Denmark). There were no changes in the indication for obtaining cultures or in the microbiological methods used for the identification of S. pneumoniae during the study period. C-reactive protein $(\mathrm{CRP})(\mathrm{mg} / \mathrm{L})$ and white blood cell count (WBC) $\left(10^{9} / \mathrm{L}\right)$, was measured at admission.

We defined GI symptoms as abdominal pain, vomiting, diarrhoea, nausea, hematemesis, melena and/or bloating. All adult patients admitted to hospital in the same period with IPD not having any GI symptoms at presentation to hospital constituted the control population. Cardiovascular disease was defined as valvular disease, cardiomegaly, coronary heart disease, arrhythmia and/or heart failure; diabetes as type 1 or type 2 diabetes mellitus; pulmonary disease as chronic obstructive pulmonary disease, tuberculosis, recurrent pneumonia or asthma; cancer as any type of malignant neoplastic disease; smoking as current smoker; alcohol abuse as drinking more than five alcohol units a day; corticosteroid use as the use of any oral or intravenous corticosteroid except inhalation; and immunosuppression as haematological cancer, corticosteroid use, cytotoxic drug treatment and asplenia.

We retrieved date of death for deceased patients within 28 days of admission to hospital from the civil registration system in Norway.

\section{Statistical methods}

We compared continuous variables using the twosample t-test, and categorical variables using Pearson's chi-squared test or Fisher's exact test.

In mortality analysis, we used univariate and multivariate Cox regression. We also graphically assessed mortality using Kaplan-Meier survival curves. Risk factors known to increase the risk of IPD were chosen as variables [14-16]. We also stratified the regression into two time periods; $1993-1999$ and 2000-2008. As this stratification reduced the number of outcomes, we had to reduce the number of variables in the regression to avoid overfitting. Therefore, we only included variables that were statistically significant in the multivariate regression for the whole period. The underlying assumptions for the regression analysis were checked and found to be adequately met. Statistical significance value was set to a $p$-value $<0.05$. Statistical analyses were performed using Stata (version 15.0 for Windows).

Ethical approval was received from the Regional Ethic Committee in Oslo.

\section{Results}

Four hundred fifty-four first time admissions for IPD were identified during the study period and included in the study. Thirty-eight patients were excluded due to incomplete data on alcohol $(n=24)$ and/or smoking $(n=$ $28)$, leaving 416 patients for the final analysis. The excluded had a higher proportion of men $(60.5 \%$ versus $44.0 \%, p=0.050$ ), but were otherwise similar to the included group (Supplement table 1).

One-hundred and eight (26.0\%) patients presented with GI symptoms, of these, $47(11.3 \%)$ presented with GI symptoms only. Compared to patients without GI symptoms, the patients with GI symptoms were younger (mean age 59.1 versus 67.1 years, $p<0.001$ ), and tended to be hospitalized for a shorter period (12.8 days versus $16.0, p=0.053)$. They had a lower proportion of cardiovascular disease $(21.3 \%$ versus $41.9 \%, \mathrm{p}<0.001)$, pulmonary disease (19.4\% versus $29.2 \%, p=0.048)$, and cancer (11.1\% versus $20.1 \%, p=0.035)$ (Table 1$)$. There were no significant differences in the occurrence of diabetes, registered smoking habits, use of corticosteroids, immunosuppression, and also no differences in registered consumption of alcohol. Similarly, we found no difference in mean CRP, or the proportion of patients with a WBC $<4$ or $>12$, at admission. When comparing the 47 patients who presented with GI symptoms only to the 
Table 1 Characteristics of 416 adult patients with invasive pneumococcal disease according to gastrointestinal symptoms at admission to Aker University Hospital between 1993 and 2008

\begin{tabular}{|c|c|c|c|c|c|c|c|c|}
\hline \multirow{2}{*}{ Age (years): } & \multicolumn{2}{|c|}{$\begin{array}{l}\text { No gastrointestinal symptoms ( } 308 \\
\text { patients) }\end{array}$} & \multicolumn{2}{|c|}{$\begin{array}{l}\text { Gastrointestinal symptoms (108 } \\
\text { patients) }\end{array}$} & \multirow[t]{2}{*}{$p^{p-v a l u e} e^{a}$} & \multicolumn{2}{|c|}{$\begin{array}{l}\text { Gastrointestinal symptoms only (47 } \\
\text { patients) }\end{array}$} & \multirow[t]{2}{*}{$p$-value $e^{a}$} \\
\hline & & & & & & & & \\
\hline Mean $(95 \% \mathrm{Cl})$ & 67.1 & $(65.2-69.0)$ & 59.1 & $(55.6-62.7)$ & $<0.001$ & 55.7 & $(50.2-61.3)$ & $<0.001$ \\
\hline Median (5-95 percentile) & 70.7 & $(33.0-89.7)$ & 58.8 & $(25.9-86.7)$ & & 56.2 & $(23.2-88.4)$ & \\
\hline \multicolumn{9}{|l|}{ Days hospitalized ${ }^{b}:$} \\
\hline Mean $(95 \% \mathrm{Cl})$ & 16.0 & $(13.9-18.2)$ & 12.8 & $(10.5-15.1)$ & 0.053 & 13.5 & $(9.7-17.3)$ & 0.192 \\
\hline Median (5-95 percentile) & 10.0 & $(1-45)$ & 9.0 & $(11-32)$ & & 10.0 & $(1-35)$ & \\
\hline Male (\%) & 137 & $(44.5)$ & 46 & $(42.6)$ & 0.734 & 18 & $(38.3)$ & 0.426 \\
\hline \multicolumn{9}{|c|}{ Inflammatory markers at admission: } \\
\hline Mean CRP $(95 \% C I)^{c}$ & 274.6 & $(256.2-293.0)$ & 290.0 & $(257.3-322.7)$ & 0.405 & 247.1 & $(205.8-288.5)$ & 0.274 \\
\hline$W B C<4$ or $>12(\%)^{d}$ & 209 & (69.9) & 72 & $(69.9)$ & 1.000 & 32 & $(71.1)$ & 0.869 \\
\hline \multicolumn{9}{|l|}{ Comorbidities (\%): } \\
\hline Cardiovascular disease & 129 & $(41.9)$ & 23 & $(21.3)$ & $<0.001$ & 9 & $(19.2)$ & 0.003 \\
\hline Pulmonary disease & 90 & $(29.2)$ & 21 & $(19.4)$ & 0.048 & 7 & $(14.9)$ & 0.040 \\
\hline Cancer & 62 & $(20.1)$ & 12 & $(11.1)$ & 0.035 & 5 & (10.6) & 0.121 \\
\hline Diabetes & 25 & (8.1) & 10 & $(9.3)$ & 0.713 & 4 & (8.5) & 1.000 \\
\hline \multicolumn{9}{|l|}{ Risk factors (\%): } \\
\hline Steroid use & 32 & $(10.4)$ & 7 & (6.5) & 0.231 & 3 & (6.4) & 0.598 \\
\hline Smoking & 127 & $(41.2)$ & 38 & $(35.2)$ & 0.269 & 19 & $(40.4)$ & 0.916 \\
\hline Alcohol & 35 & (11.4) & 11 & $(10.2)$ & 0.737 & 6 & (12.8) & 0.779 \\
\hline Immunosuppressed $^{\mathrm{e}}$ & 48 & (15.6) & 11 & $(10.2)$ & 0.166 & 4 & (8.5) & 0.269 \\
\hline
\end{tabular}

acompared to no gastrointestinal symptoms

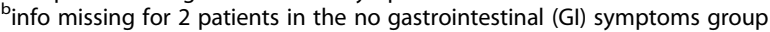

info missing for 17 patients in the no Gl symptoms, 5 in the Gl symptoms, and 2 in the only Gl symptoms group

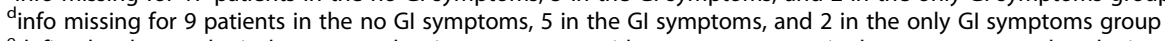

edefined as hematological cancer, oral or intravenous steroid treatment, cytotoxic drug treatment and asplenia

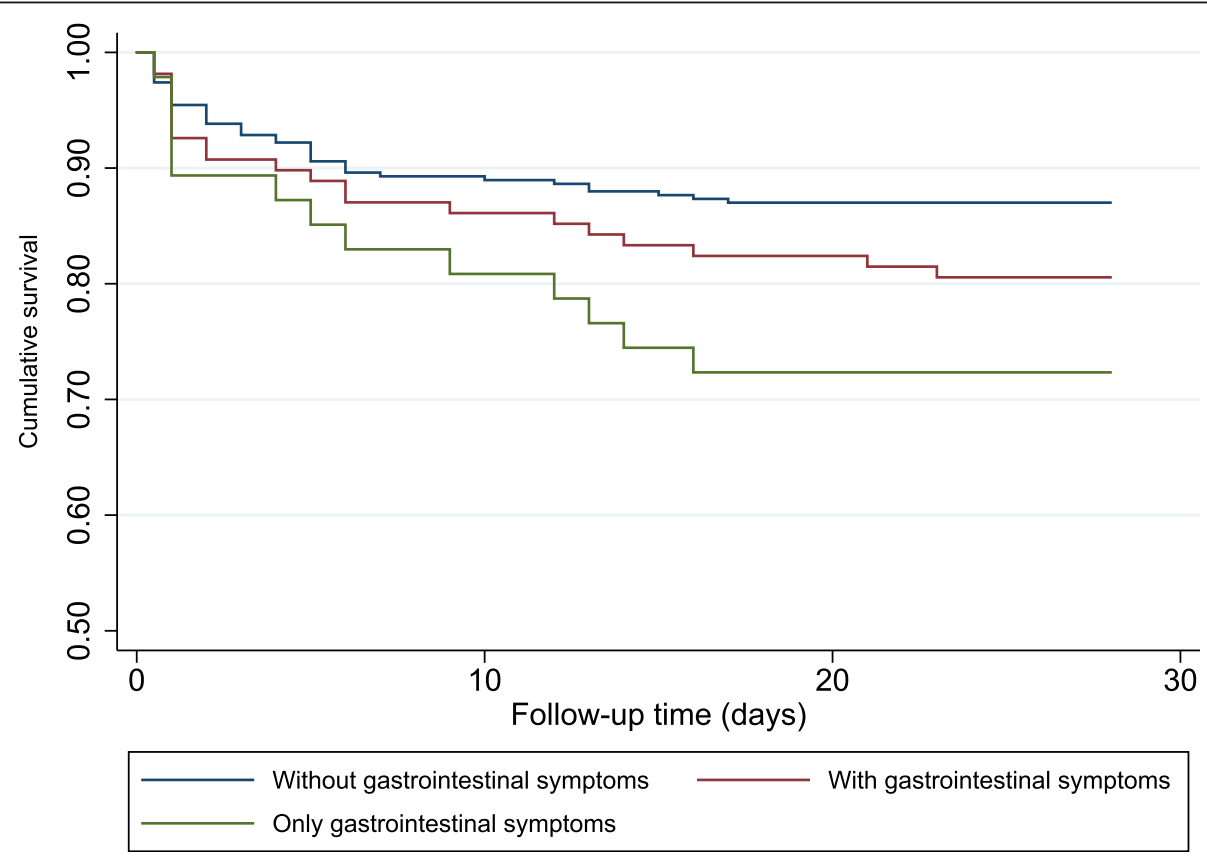

Fig. 1 Kaplan-Meier survival curve of adult patients with invasive pneumococcal disease stratified by symptoms at admission to Aker University Hospital, Oslo, Norway, between 1993 and 2008 
group without GI symptoms, we found a similar trend, with the patients without GI symptoms on average being older and having more comorbidities.

Sixty-one patients (14.7\% of the total study population) died within 28 days of hospital admission. Of the 308 patients without GI symptoms, 40 died (13.0\%). Of the 108 patients with GI symptoms, 21 died (19.4\%), and 13 of the 47 patients (27.7\%) with GI symptoms only died within 28 days. Kaplan-Meier survival curves show an increased 28-day mortality in the group of patients presenting with GI symptoms (Fig. 1). Univariate Cox regression showed that patients presenting with GI symptoms had a non-significantly increased hazard ratio of 1.53 (95\% CI 0.90-2.59) (Table 2). However, adjusting for the known risk factors, we found that having GI symptoms upon presentation of IPD significantly predicted death within 28 days of hospital admission, with a hazard ratio of 2.28 (95\% CI 1.31-3.97). When stratifying by time periods, this statistically increased mortality was only noted in the early time period with a hazard ratio of 3.93 (95\% CI 1.75-8.82) (Supplement table 4). The patients presenting with GI symptoms only had a significantly higher hazard ratio of 2.24 (95\% CI 1.20 $4.19)$ in univariate analysis, and 4.20 (95\% CI 2.11-8.39) in the multivariate analysis (Table 3). In this group, there was no difference in the hazard ratio between the two time periods (Supplement table 4).

In most patients, the focus of infection was assumed to be the lungs. In the group that presented with GI symptoms, a larger proportion was discharged with an unknown focus of infection (Table 4).

All the pneumococcal isolates were penicillinsusceptible. Pneumococcal serotype was identified in $301(72.4 \%)$ of the 416 samples (Supplement table 2). Serotype 1 was the most common in the group presenting with GI symptoms, accounting for $26.5 \%$ of the typeable serotypes in patients presenting with gastrointestinal symptoms and $33.3 \%$ in patients presenting with GI symptoms only. Serotype 1 was less prevalent in patients not presenting with gastrointestinal

Table 2 Univariate and multivariate survival analysis by Cox regression for 416 adult patients with invasive pneumococcal disease at admission to Aker University Hospital between 1993 and 2008

\begin{tabular}{|c|c|c|c|c|c|}
\hline \multicolumn{6}{|l|}{ Univariate analysis } \\
\hline Variables & Hazard ratio & Std.err & $p$-value & $95 \% \mathrm{Cl}$ & \\
\hline Gastrointestinal symptoms & 1.53 & 0.41 & 0.116 & 0.90 & -2.59 \\
\hline Age & 1.03 & 0.01 & $<0.001$ & 1.02 & -1.05 \\
\hline Being female & 0.60 & 0.15 & 0.046 & 0.36 & -0.99 \\
\hline Cardiovascular disease & 1.62 & 0.41 & 0.061 & 0.98 & -2.67 \\
\hline Diabetes & 1.68 & 0.64 & 0.169 & 0.80 & -3.54 \\
\hline Pulmonary disease & 1.36 & 0.37 & 0.255 & 0.80 & -2.33 \\
\hline Cancer & 1.73 & 0.50 & 0.061 & 0.98 & -3.06 \\
\hline Alcohol & 1.53 & 0.53 & 0.222 & 0.77 & -3.01 \\
\hline Smoking & 1.13 & 0.29 & 0.626 & 0.68 & -1.88 \\
\hline Steroid & 1.74 & 0.63 & 0.126 & 0.86 & -3.52 \\
\hline Antibiotics given immediately after blood culture was drawn & 0.49 & 0.25 & 0.170 & 0.18 & -1.36 \\
\hline \multicolumn{6}{|l|}{ Multivariate analysis } \\
\hline Variables & Hazard ratio & Std.err & p-value & $95 \% \mathrm{Cl}$ & \\
\hline Gastrointestinal symptoms & 2.22 & 0.63 & 0.005 & 1.27 & -3.89 \\
\hline Age & 1.05 & 0.01 & 0.000 & 1.03 & -1.07 \\
\hline Being female & 0.52 & 0.14 & 0.016 & 0.31 & -0.88 \\
\hline Cardiovascular disease & 1.03 & 0.30 & 0.905 & 0.59 & -1.82 \\
\hline Diabetes & 1.56 & 0.62 & 0.259 & 0.72 & -3.38 \\
\hline Pulmonary disease & 0.98 & 0.29 & 0.955 & 0.55 & -1.76 \\
\hline Cancer & 1.58 & 0.50 & 0.142 & 0.86 & -2.93 \\
\hline Alcohol & 1.89 & 0.72 & 0.098 & 0.89 & -4.00 \\
\hline Smoking & 1.35 & 0.38 & 0.284 & 0.78 & -2.33 \\
\hline Steroid & 1.58 & 0.59 & 0.226 & 0.75 & -3.29 \\
\hline Antibiotics given immediately after blood culture was drawn & 0.62 & 0.34 & 0.381 & 0.22 & -1.79 \\
\hline
\end{tabular}


Table 3 Univariate and multivariate survival analysis by Cox regression for 355 adult patients with invasive pneumococcal disease at admission to Aker University Hospital between 1993 and 2008

\begin{tabular}{|c|c|c|c|c|c|}
\hline \multicolumn{6}{|l|}{ Univariate analysis } \\
\hline Variable & Hazard ratio & Std.err & $p$-value & $95 \% \mathrm{Cl}$ & \\
\hline Gastrointestinal symptoms only & 2.24 & 0.72 & 0.011 & 1.20 & -4.19 \\
\hline Age & 1.03 & 0.01 & 0.001 & 1.01 & -1.05 \\
\hline Being female & 0.66 & 0.18 & 0.136 & 0.39 & -1.14 \\
\hline Cardiovascular disease & 1.43 & 0.39 & 0.192 & 0.83 & -2.45 \\
\hline Diabetes & 1.74 & 0.71 & 0.173 & 0.79 & -3.85 \\
\hline Pulmonary disease & 1.54 & 0.44 & 0.135 & 0.88 & -2.69 \\
\hline Cancer & 1.45 & 0.46 & 0.247 & 0.77 & -2.71 \\
\hline Alcohol & 1.32 & 0.51 & 0.473 & 0.62 & -2.79 \\
\hline Smoking & 1.21 & 0.33 & 0.499 & 0.70 & -2.07 \\
\hline Steroid & 1.40 & 0.57 & 0.407 & 0.63 & -3.10 \\
\hline Antibiotics given immediately after blood culture was drawn & 0.46 & 0.24 & 0.134 & 0.17 & -1.27 \\
\hline \multicolumn{6}{|l|}{ Multivariate analysis } \\
\hline Variable & Hazard ratio & Std.err & p-value & $95 \% \mathrm{Cl}$ & \\
\hline Gastrointestinal symptoms only & 3.96 & 1.43 & 0.000 & 1.95 & -8.06 \\
\hline Age & 1.05 & 0.01 & 0.000 & 1.03 & -1.07 \\
\hline Being female & 0.49 & 0.14 & 0.015 & 0.27 & -0.87 \\
\hline Cardiovascular disease & 1.00 & 0.31 & 0.998 & 0.55 & -1.83 \\
\hline Diabetes & 1.56 & 0.67 & 0.303 & 0.67 & -3.61 \\
\hline Pulmonary disease & 1.34 & 0.43 & 0.369 & 0.71 & -2.51 \\
\hline Cancer & 1.39 & 0.48 & 0.342 & 0.70 & -2.74 \\
\hline Alcohol & 1.53 & 0.65 & 0.315 & 0.67 & -3.53 \\
\hline Smoking & 1.34 & 0.42 & 0.344 & 0.73 & -2.46 \\
\hline Steroid & 1.23 & 0.52 & 0.623 & 0.54 & -2.84 \\
\hline Antibiotics given immediately after blood culture was drawn & 0.63 & 0.36 & 0.419 & 0.20 & -1.95 \\
\hline
\end{tabular}

symptoms $(15.1 \%$ vs 26.5 and $33.3 \%, p=0.023$ and $p=$ 0.014).

Empirical antimicrobial treatment varied between the groups. There was less use of monotherapy with penicillin in the presence of GI symptoms (32\% versus $46 \%$, $p=0.013)$. Also, there was more use of other antibiotics than the combination of penicillin and gentamicin in patients presenting with GI symptoms only compared to no GI symptoms (43\% versus $22 \%, p<0.001$ ) (Supplement table 3). Furthermore, there was a significant difference between the groups in whether or not they received antibiotics immediately after blood culture was drawn. While only $3 \%$ of those without GI symptoms received no antibiotics after blood culture was drawn,

Table 4 Focus of invasive pneumococcal disease stratified by symptoms for adult patients admitted to Aker University Hospital between 1993 and 2008

\begin{tabular}{|c|c|c|c|c|c|c|}
\hline \multirow[t]{2}{*}{ Focus at discharge } & \multicolumn{2}{|c|}{ No gastrointestinal symptoms } & \multicolumn{2}{|c|}{ Gastrointestinal symptoms } & \multicolumn{2}{|c|}{ Gastrointestinal symptoms only } \\
\hline & $n$ & (\%) & $n$ & (\%) & $n$ & (\%) \\
\hline Lower respiratory tract & 273 & 89 & 87 & 80 & 31 & 66 \\
\hline Abdominal & 0 & 0 & 1 & 1 & 0 & 0 \\
\hline Central nervous system & 8 & 3 & 5 & 5 & 4 & 8 \\
\hline Other & 7 & 2 & 1 & 1 & 0 & 0 \\
\hline \multirow[t]{2}{*}{ Unknown } & 20 & 6 & 14 & 13 & 12 & 26 \\
\hline & 308 & & 108 & & 47 & \\
\hline
\end{tabular}


$13 \%$ of those with GI symptoms only did not $(p=0.001)$. Of the 15 patients that received no antibiotic treatment after blood culture was drawn, ten received penicillin after the blood culture results were available. Among the last five, two were transferred to another hospital within 1 day, and three died within 1, 3 or 7 days, respectively. Including whether antibiotics were given or not immediately after blood culture as a variable in the Cox regression models did not affect the results.

\section{Discussion}

In this study, we found that more than a quarter of patients with IPD presented with GI symptoms at admission. These patients had a higher short-term mortality compared to patients without GI symptoms, despite being younger and having less comorbidities. The increased mortality became even more apparent when restricting the group to those who presented with GI symptoms only. Furthermore, we found that there was a delay in the initiation of adequate antibiotic treatment in the patients presenting with GI symptoms.

\section{Interpretation}

Few studies have investigated GI symptoms in IPD. Watanakunakorn and Bailey found that $10.2 \%$ of patients with bacteremic pneumococcal pneumonia had abdominal pain at presentation to the hospital [6]. Their study population is comparable to ours regarding age, comorbidity and mortality, but importantly, only patients with evidence of pneumonia were included in that study, and no characterization of the patients with abdominal pain was given. Weiss et al. found that $33.6 \%$ of patients with bacteremic pneumococcal pneumonias had nausea and vomiting necessitating antiemetic treatment or preventing them from eating [7]. Weiss' study included only bacteremic pneumonias and excluded infectious complications like meningitis, abscesses, empyema, endocarditis and septic arthritis. The study is otherwise comparable to our study, although a further characterization of patients who had nausea and vomiting at admission was not given.

Some studies are supporting our finding that IPD patients with GI symptoms have a higher short-term mortality. In a small study of 38 patients admitted to an intensive care unit with severe pneumococcal bacteremia [17], the in-hospital mortality rate was $89 \%$ for the nine patients with GI symptoms and $24 \%$ for the 29 patients without gastrointestinal symptoms, i.e. a 3.7 times higher mortality. Although these numbers have to be interpreted with caution due to small sample size, this increased mortality risk is similar to our findings. Another study of 289 patients with unspecified communityacquired infections, found that patients with diarrhoea or vomiting at admission had an increased risk of developing severe sepsis within $48 \mathrm{~h}$ of admission [18]. This study might suggest that GI symptoms are not only a marker of poor outcome in IPD but a marker of poor outcome of sepsis in general. However, this study had only 26 positive blood cultures and included all possible foci of infection and microbial agents. Therefore, this study is not directly comparable to ours.

Another explanation for the higher mortality in patients with GI symptoms might be that it could blur the diagnostic evaluation and lead to delayed initiation of adequate therapy. A large proportion of the group with GI symptoms had a focus of unknown origin, which may prolong the time to diagnosis and treatment. More patients with GI symptoms only received no antibiotics early after blood cultures were taken, and if they did, more patients received other antibiotics than penicillin and gentamicin. We do not have data on the exact time to start of antimicrobial therapy in this study, but we know that a reduced time to treatment improves outcome [19]. It is also possible that these patients had an undiagnosed abdominal focus, which could have delayed the initiation of appropriate antibiotic therapy. Several case reports and case series have previously reported abdominal or gynaecological focuses in pneumococcal infections [8-10].

The decision to perform a spinal tap was based on clinical suspicion of meningitis. If there were meningitis cases present misdiagnosed as pneumonia or unknown focus, this might have contributed to an increased mortality in the GI symptoms group as nausea is a common symptom in bacterial meningitis [20]. However, as this was a population with no pneumococcal isolates resistant to penicillin, patients with undiagnosed meningitis would still have been adequately treated with penicillin [21].

In our study, serotype 1 was more prevalent in patients with GI symptoms, and even more prevalent in patients with GI symptoms only. Other studies have found that patients infected by serotype 1 tend to be younger and without significant comorbidity [22-24]. This is in agreement with our finding of less comorbidity in patients with GI symptoms. However, it does not explain the higher mortality, as serotype 1 previously has been associated with a lower mortality rate compared to other serotypes [25].

According to our regression analysis, females had a lower short-term mortality compared to men. When stratifying our analysis into the two time periods, we found that this difference was only significant during the first 7 years. Almost $28 \%$ of men died within 28 days in the period 1993-1999, compared to only $11 \%$ of women in the same period (supplement table 5). We do not have a good explanation for this increased mortality in men during the first 7 years. There is conflicting 
evidence in previous studies. For instance, while a higher mortality was found in men than in women with pneumococcal bacteremia [26], a study of severe sepsis and septic shock showed higher mortality in women compared to men [27].

\section{Limitations}

Although it is a strength of the present study that it is a large study of unselected IPD patients with a 16-year long observation time, we did not have complete data on clinical characteristics. Respiratory rate (RR) could have been used to test the association between GI symptoms and severity of sepsis as assessed by using CRB-65, SIRS, SOFA and q-SOFA, and GI symptoms, but RR was only reported in about $40 \%$ of the patients. However, we found no difference in inflammatory markers at admission. A second limitation is that data on concurrent severe GI disease and complications were not registered. Gastrointestinal bleeding and perforation are examples of conditions that possibly could have contributed to the increased mortality in the GI symptoms group. Another limitation is the retrospective registration of data using medical records to assess subjective symptoms. However, as the data was collected using predefined forms, we do not believe that this introduced any nondifferential measurement bias.

\section{Conclusions}

A large proportion of IPD presents with GI symptoms combined with other symptoms or alone. GI symptoms in IPD is associated with a significantly higher shortterm mortality and delayed treatment. It is important to be aware of this atypical presentation of IPD in order to initiate proper treatment rapidly.

\section{Supplementary information}

Supplementary information accompanies this paper at https://doi.org/10. 1186/s12879-020-05211-3.

\section{Additional file 1 : Table S1. Characteristics of included and excluded adult patients with invasive pneumococcal disease at admission to Aker University Hospital between 1993 and 2008. Table S2. Serotype of invasive pneumococcal disease stratified by symptoms for 416 adult patients admitted to Aker University Hospital between 1993 and 2008. Table S3. Empirical antibiotic treatment initiated after blood culture in 416 adult patients with invasive pneumococcal disease admitted to Aker University Hospital between 1993 and 2008. n:number. Table S4. \\ Multivariate survival analysis by Cox regression for 416 adult patients with invasive pneumococcal disease at admission to Aker University Hospital between 1993 and 2008 by time period. Gl: Gastrointestinal. Table S5. Proportion dead within 28 days for 416 adult patients with invasive pneumococcal disease at admission to Aker University Hospital between 1993 and 2008 by sex and time period.}

\section{Abbreviations}

IPD: Invasive pneumococcal disease; GI: Gastrointestinal; AUH: Aker University Hospital; NIPH: Norwegian Institute of Public Health; Cl: Confidence interval; RR: Respiratory rate

\section{Acknowledgements}

Leiv Sandvik contributed to the statistical analysis of the data and revision of the paper. Kristine Schiøtz, Elisabeth Lie and Jonas Lian contributed by registration of patient data.

\section{Authors' contributions}

HKF has contributed to the conception and design of the study, acquisition, analysis and interpretation of the data, drafting and revision of the manuscript. JDB has contributed to the conception and design of the study, analysis and interpretation of the data, and drafting and revision of the manuscript. BJB has contributed to acquisition of data and revising the manuscript. DFV has contributed to the design of the study, acquisition and interpretation of the data, and revising of the manuscript. DB has contributed to the conception and design of the study, acquisition, analysis and interpretation of the data, and drafting and revision of the manuscript.

$\mathrm{AMH}$ has contributed to the conception and design of the study, acquisition of data, and revising of the manuscript. All authors approved the final version to be submitted.

\section{Funding}

No external funding was received.

\section{Availability of data and materials}

The data that support the findings of this study are available from the authors upon reasonable request.

\section{Ethics approval and consent to participate}

Ethics approval was received from the Regional Ethic Committee in Oslo, South-Eastern Norway, before the study started in 1993. As there was no Regional Ethic Committee register of projects at the time, there is no reference number. The study was re-approved by the director of the Regional Ethic Committee in South-Eastern Norway in 2015, Knut W. Ruyter.

Consent for publication

Not applicable.

Competing interests

The authors declare that they have no competing interests.

\section{Author details}

'Department of Internal Medicine, Sorlandet Hospital, Kristiansand, Norway. ${ }^{2}$ Norwegian Institute of Public Health, Division of infection control and environmental health, Oslo, Norway. ${ }^{3}$ Department of Internal Medicine, Lovisenberg Hospital, Oslo, Norway. ${ }^{4}$ Department of Infectious Disease, Oslo University Hospital, Oslo, Norway. ${ }^{5}$ Institute of Clinical Medicine, Faculty of Medicine, University of Oslo, Oslo, Norway. ${ }^{6}$ Department of Respiratory Medicine, Oslo University Hospital, Oslo, Norway.

Received: 1 March 2020 Accepted: 29 June 2020

Published online: 06 July 2020

\section{References}

1. Jain $S$, Self WH, Wunderink RG, Fakhran S, Balk R, Bramley AM, et al. Community-acquired pneumonia requiring hospitalization among U.S. adults. N Engl J Med. 2015;373(5):415-27.

2. Johansson N, Kalin M, Tiveljung-Lindell A, Giske CG, Hedlund J. Etiology of community-acquired pneumonia: increased microbiological yield with new diagnostic methods. Clin Infect Dis. 2010:50(2):202-9.

3. Drijkoningen JJ, Rohde GG. Pneumococcal infection in adults: burden of disease. Clin Microbiol Infect. 2014;20(Suppl 5):45-51.

4. Floeystad HK, Holm A, Sandvik L, Vestrheim DF, Brandsaeter B, Berild D. Increased long-term mortality after survival of invasive pneumococcal disease: a population-based study. Infect Dis. 2017:49(5):365-72.

5. Yu VL, Chiou CC, Feldman C, Ortqvist A, Rello J, Morris AJ, et al. An international prospective study of pneumococcal bacteremia: correlation with in vitro resistance, antibiotics administered, and clinical outcome. Clin Infect Dis. 2003;37(2):230-7.

6. Watanakunakorn C, Bailey TA. Adult bacteremic pneumococcal pneumonia in a community teaching hospital, 1992-1996. A detailed analysis of 108 cases. Arch Intern Med. 1997;157(17):1965-71. 
7. Weiss K, Low DE, Cortes L, Beaupre A, Gauthier R, Gregoire P, et al. Clinical characteristics at initial presentation and impact of dual therapy on the outcome of bacteremic Streptococcus pneumoniae pneumonia in adults. Can Respir J. 2004;11(8):589-93.

8. Bucher A, Muller F. Spectrum of abdominal and pelvic infections caused by pneumococci in previously healthy adult women. Eur I Clin Microbiol Infect Dis. 2002;21(6):474-7.

9. Capdevila O, Pallares R, Grau I, Tubau F, Linares J, Ariza J, et al. Pneumococcal peritonitis in adult patients: report of 64 cases with special reference to emergence of antibiotic resistance. Arch Intern Med. 2001; 161(14):1742-8.

10. Hemsley C, Eykyn SJ. Pneumococcal peritonitis in previously healthy adults: case report and review. Clin Infect Dis. 1998;27(2):376-9.

11. Song JY, Nahm MH, Moseley MA. Clinical implications of pneumococcal serotypes: invasive disease potential, clinical presentations, and antibiotic resistance. J Korean Med Sci. 2013;28(1):4-15.

12. Balakrishnan I, Crook P, Morris R, Gillespie SH. Early predictors of mortality in pneumococcal bacteraemia. J Infect. 2000;40(3):256-61.

13. Gransden WR, Eykyn SJ, Phillips I. Pneumococcal bacteraemia: 325 episodes diagnosed at St Thomas's hospital. Br Med J (Clin Res Ed). 1985;290(6467): 505-8.

14. Cruickshank HC, Jefferies JM, Clarke SC. Lifestyle risk factors for invasive pneumococcal disease: a systematic review. BMJ Open. 2014;4(6):e005224.

15. Torres A, Blasi F, Dartois N, Akova M. Which individuals are at increased risk of pneumococcal disease and why? Impact of COPD, asthma, smoking, diabetes, and/or chronic heart disease on community-acquired pneumonia and invasive pneumococcal disease. Thorax. 2015;70(10):984-9.

16. van Hoek AJ, Andrews N, Waight PA, Stowe J, Gates P, George R, et al. The effect of underlying clinical conditions on the risk of developing invasive pneumococcal disease in England. J Infect. 2012;65(1):17-24.

17. Ursin Rein P, Jacobsen D, Ormaasen V, Dunlop O. Pneumococcal sepsis requiring mechanical ventilation: cohort study in 38 patients with rapid progression to septic shock. Acta Anaesthesiol Scand. 2018;62(10):1428-35.

18. Edman-Waller J, Ljungstrom L, Jacobsson G, Andersson R, Werner M. Systemic symptoms predict presence or development of severe sepsis and septic shock. Infect Dis. 2016;48(3):209-14.

19. Seymour CW, Gesten F, Prescott HC, Friedrich ME, Iwashyna TJ, Phillips GS, et al. Time to treatment and mortality during mandated emergency Care for Sepsis. N Engl J Med. 2017;376(23):2235-44.

20. van de Beek D, de Gans J, Spanjaard L, Weisfelt M, Reitsma JB, Vermeulen M. Clinical features and prognostic factors in adults with bacterial meningitis. N Engl J Med. 2004;351(18):1849-59.

21. Brouwer MC, Tunkel AR, van de Beek D. Epidemiology, diagnosis, and antimicrobial treatment of acute bacterial meningitis. Clin Microbiol Rev. 2010;23(3):467-92.

22. Ritchie ND, Mitchell TJ, Evans TJ. What is different about serotype 1 pneumococci? Future Microbiol. 2012;7(1):33-46

23. Austrian R, Gold J. Pneumococcal bacteremia with especial reference to BACTEREMIC pneumococcal pneumonia. Ann Intern Med. 1964;60:759-76.

24. Harboe ZB, Thomsen RW, Riis A, Valentiner-Branth P, Christensen JJ, Lambertsen $L$, et al. Pneumococcal serotypes and mortality following invasive pneumococcal disease: a population-based cohort study. PLoS Med. 2009;6(5):e1000081.

25. Weinberger DM, Harboe ZB, Sanders EA, Ndiritu M, Klugman KP, Ruckinger $S$, et al. Association of serotype with risk of death due to pneumococcal pneumonia: a meta-analysis. Clin Infect Dis. 2010;51(6):692-9.

26. Cobo F, Cabezas-Fernández MT, Cabeza-Barrera MI. Streptococcus pneumoniae bacteremia: clinical and microbiological epidemiology in a health area of Southern Spain. Infect Dis Rep. 2012:4(2):e29-e.

27. Pietropaoli AP, Glance LG, Oakes D, Fisher SG. Gender differences in mortality in patients with severe sepsis or septic shock. Gend Med. 2010; 7(5):422-37.

\section{Publisher's Note}

Springer Nature remains neutral with regard to jurisdictional claims in published maps and institutional affiliations.

\section{Ready to submit your research? Choose BMC and benefit from:}

- fast, convenient online submission

- thorough peer review by experienced researchers in your field

- rapid publication on acceptance

- support for research data, including large and complex data types

- gold Open Access which fosters wider collaboration and increased citations

- maximum visibility for your research: over $100 \mathrm{M}$ website views per year

At BMC, research is always in progress.

Learn more biomedcentral.com/submissions 